\title{
Validación de un método de análisis para la determinación de hidrocarburos aromáticos policíclicos por cromatografía líquida de alta eficiencia en partículas PMIOY PM2,5
}

\author{
Validation of an analytical method for \\ the determination of polycyclic aromatic \\ hydrocarbons by high efficiency liquid \\ chromatography in PMIO and PM2, 5
}

Jorge Herrera Murillo' María del Carmen Chaves Villalobos ${ }^{2}$

Fecha de recepción: 13 de junio del 2011 Fecha de aprobación: 9 de marzo del 2012

Herrera, J; Chaves, M. Validación de un método de análisis para la determinación de hidrocarburos aromáticos policíclicos por cromatografía líquida de alta eficiencia en partículas PMIO Y PM2,5. Tecnología en Marcha. Vol. 25, No 3. Julio-Setiembre 2012. Pág 39-49.

Químico. Laboratorio de Análisis Ambiental, Universidad Nacional. Teléfono: 2277-3292 Correo electrónico: jherrer@una.ac.cr

2. Química industrial. Laboratorio de Análisis Ambiental, Universidad Nacional Teléfono:

Correo electrónico: maria.chaves.villalobos@una.cr 


\section{Resumen}

Se validó un método analítico para la determinación de hidrocarburos aromáticos policíclicos presentes en partículas PMIO y PM 2,5 recolectadas en el aire mediante cromatografía líquida de alta resolución (CLAR).

Los HPA incluidos en la metodología comprenden: Naftaleno, Acenaftileno, Fluoreno, Acenafteno, Fenantreno, Antraceno, Fluoranteno, Pireno, Benzo (a)antraceno, Criseno, Benzo (b)fluoranteno, Benzo (k)fluoranteno, Benzo (a)pireno, Dibenzo $(a, h)$ antraceno, Benzo (g,h,i)perileno y Indeno (I,2,3C.D) pireno.

Para estos compuestos, los límites de detección y cuantificación estuvieron entre 0,02 y 0,I mg/l utilizando un equipo marca Dionex modelo ICS3000, el cual consta de dos detectores en serie, un ultravioleta modelo WWD-I y un detector de fluorescencia modelo RF-2000, permitiendo diferenciar las distintas señales de absorción y emisión para la debida identificación de los distintos compuestos.

Para todos los compuestos analizados, el factor de recuperación resultó no ser significativamente diferente de uno y la repetibilidad y reproducibilidad resultó ser adecuada para un método analítico, especialmente para los HPA más ligeros.

\section{Palabras clave}

Validación, cromatografía líquida de alta eficiencia, hidrocarburos aromáticos policíclicos, partículas.

\section{Abstract}

An analytical method for polycyclic aromatic hydrocarbons in PMI 0 and PM 2.5 particles collected from air by high performance liquid chromatography (HPLC) was validated.

The PAHs analyzed in the methodology include: Naphthalene, Acenaphthylene, Fluorene, Acenaphthene, Phenanthrene, Anthracene, fluoranthene, pyrene, Benzo (a)anthracene, Chrysene, Benzo (b)fluoranthene, Benzo (k)fluoranthene, Benzo (a)pyrene Dibenzo (a, h)anthracene, Benzo (g, h, i)perylene and Indeno (I,2,3-CD)pyrene.

For these compounds, the detection limit and quantification limit were between 0,02 and 0,1 mg/l using a DIONEX ICS 3000 model cromatograph, that has two in serie detectors: UVNis and Fluorescense, separating the different absorption and emission signals for proper identification of individual compounds.

For all the compounds analyzed the recovery factor was not significantly different from each other and the repeatability and reproducibility was found to be suitable for an analytical method, especially for the lighter PAHs.

\section{Key words}

Validation, high performance liquid cromatography, policiclic aromatic hydrocarbons, particles. 


\section{Introducción}

La contaminación atmosférica puede definirse como la introducción a la atmósfera de sustancias o energía que alteran su composición natural, afectando su funcionamiento normal. Estos contaminantes atmosféricos se pueden clasificar en contaminantes criterio y contaminantes no criterio. Los primeros han sido identificados como comunes y perjudiciales para la salud y el bienestar del ser humano.

Las partículas son uno de los contaminantes criterio que generan más problemas en la actualidad y se clasifican tomando en cuenta su diámetro aerodinámico, que puede variar en el caso de las partículas suspendidas totales (PST) desde los 0,00 I $\mu \mathrm{m}$ hasta los $100 \mu \mathrm{m}$ (Saldarriaga, 2008).

Las diferencias en la toxicidad de las partículas se pueden asociar no solo al incremento en su concentración másica, sino también a las variaciones en el tamaño de estas (Schwartz y Meas, 2000), su forma y química (Richards, 1997, Adamson et al., 2000) y a la presencia de pequeñas cantidades de elementos altamente tóxicos (Nawrot et al., 2006), especialmente si estos son solubles en agua (Fernández Espinosa et al., 2002, Birmili et al., 2006). En este contexto, los hidrocarburos aromáticos policíclicos, presentes en las partículas generalmente en pequeñas cantidades, juegan un papel muy importante en los problemas de salud humana.

Por definición, un hidrocarburo aromático policíclico (HAP) consta de dos o más anillos bencénicos, en posición lineal, angular o con un conjunto de arreglos, y están compuestos únicamente de carbono e hidrógeno (Seinfeld y Pandis, 1998). Los HAP mantienen variable su peso molecular, dependiendo del número de anillos que contengan. Los de bajo peso molecular tienden a estar más concentrados en la fase de vapor, mientras que los de mayor peso molecular se asocian a menudo con partículas (Sheng et al., 2003).

La formación de estos compuestos está dada por pirólisis o combustión incompleta de materia orgánica que contiene carbono e hidrógeno. Cuando la pirólisis de compuestos ocurre a temperaturas muy altas, se producen fragmentos de moléculas y radicales que se combinan para dar lugar a la formación de los HAP.

La composición de estos dependerá en su totalidad del tipo de combustible, la temperatura y el tiempo de residencia del compuesto a dicha temperatura. Dentro de los combustibles que forman HAP se encuentran: metano, otros hidrocarburos, hidratos de carbono, ligninas y péptidos, entre otros (Mastandrea et al., 2005).

También existen otra clase de compuestos insaturados y algunas estructuras cíclicas que favorecen la formación de estos compuestos. Los HAP se liberan en forma de gas, en la fuente de emisión, y por lo general se condensan de forma inmediata sobre las partículas de hollín; también mantienen la capacidad de formar ellos mismos partículas muy pequeñas.

Las partículas que se encuentran en la atmósfera son capaces de absorber los HAP que son liberados como vapor, generando con esto una diseminación de aerosoles que contienen HAP, los cuales por influencia de factores fisicoquímicos, como los vientos, pueden ser transportados a distancias muy largas (Mastandrea et al., 2005).

Se ha encontrado que en la atmósfera, los HAP se producen por fuentes naturales $O$ antropogénicas, como las emisiones provenientes de un motor de escape, las emisiones industriales, la incineración de basura, la quema de combustibles fósiles en procesos energéticos y la quema de biomasa (Figuren y Miguel, 2003, Ravindra et al., 2006).

En el caso de las fuentes biogénicas, se mantienen desprendimientos de las ceras epicuticulares de plantas y la suspensión directa de polen por microorganismos e insectos (Cincinelli et al., 2007, Sheng et al., 2003), incendios forestales y erupciones volcánicas (Seinfeld y Pandis, 1998).

La estabilidad química de los HAP viene dada por los sistemas conjugados $\varpi$, cuando están a temperatura ambiente, se encuentran en estado sólido y con poca volatilidad. Estos compuestos absorben luz ultravioleta y producen un espectro fluorescente de acuerdo con su carácter aromático.

Presentan solubilidad variada en solventes orgánicos pero son prácticamente insolubles en agua, conforme mayor sea su peso molecular. Las reacciones químicas que estos compuestos presentan son la sustitución de hidrógeno o la adición cuando se produce la saturación, manteniendo siempre el arreglo de anillos. 
Según estudios toxicológicos, no todos los HAP presentan actividad carcinogénica, genotóxica o mutagénica; en la mayoría de los casos, este efecto se le atribuye a la presencia en conjunto de un compuesto perteneciente a la familia y algunos de sus derivados, en especial los nitroderivados (Stellman y McCan, 1998).

Los estudios han arrojado datos que llevan a la conclusión de que los HAP son activados previamente en el cuerpo, antes de funcionar como perturbadores endocrinos, cancerígenos o mutagénicos. La exposición prolongada a estos compuestos puede producir cáncer de escroto, cara, broncogénico y de vejiga, además de leucemia y linfoma (Mastandrea et al., 2005).

En el presente artículo se exponen los resultados del proceso de validación de un método analítico empleado en la determinación del contenido de hidrocarburos aromáticos policíclicos en partículas $P M_{10}$ y $P M_{2,5}$.

\section{Metodología}

\section{Tratamiento de la cristalería}

La cristalería se lavó de forma minuciosa, realizando enjuagues al final con agua mili-Q y acetona grado HPLC, para su posterior secado en estufa a 110 ${ }^{\circ} \mathrm{C}$ por cuatro horas, esto eliminó los compuestos orgánicos que pudieron quedar luego de los lavados correspondientes. El uso de papel aluminio ayudó a la conservación de los estándares, ya que presentan sensibilidad a la luz fluorescente, además de evitar el contacto con el polvo.

\section{Patrones de calibración}

La curva de calibración se preparó a partir de una disolución estándar certificada que contenía 16 hidrocarburos aromáticos policíclicos con una concentración de 200 mg/l, marca CHEM SERVICE con número de catálogo PNA-550RPM. Esta disolución contempla los siguientes compuestos: Naftaleno, Acenaftileno, Fluoreno, Acenafteno, Fenantreno, Antraceno, Fluoranteno, Pireno, Benzo (a) antraceno, Criseno, Benzo (b) fluoranteno, Benzo (k) fluoranteno, Benzo (a) pireno, Dibenzo $(a, h)$ antraceno, Benzo (g,h,i) perileno e Indeno (I,2,3C.D) pireno.

La preparación de las disoluciones de la curva de calibración se logró mediante una solución intermedia de 5 mg/l, que contenía los 16 HAP que se deseaban analizar. A partir de esta disolución intermedia, se tomaron alícuotas desde 0,02 a 0,200 $\mathrm{ml}$ con pipetas graduadas de vidrio, evitando el uso de micropipetas con puntas de plástico, ya que este material interfiere en el análisis de HPA. En todos los casos se utilizó acetonitrilo como disolvente para realizar las diluciones.

\section{Cuadro I. Condiciones instrumentales para la determinación de HPA}

\begin{tabular}{|c|c|c|c|}
\hline Flujo de fase móvil & \multicolumn{3}{|c|}{$\mathrm{I} \mathrm{ml} / \mathrm{min}$} \\
\hline \multirow{4}{*}{$\begin{array}{l}\text { Gradiente de fase móvil, con } \\
\text { rampa lineal }\end{array}$} & Tiempo (min) & $\%$ Acetonitrilo & \% Agua desionizada \\
\hline & 0 & 60 & 40 \\
\hline & 20 & 100 & 0 \\
\hline & 35 & 60 & 40 \\
\hline Temperatura de la columna & \multicolumn{3}{|c|}{$20.00^{\circ} \mathrm{C}$} \\
\hline $\begin{array}{l}\text { Temperatura del } \\
\text { automuestreador }\end{array}$ & \multicolumn{3}{|c|}{$20,00^{\circ} \mathrm{C}$} \\
\hline Volumen de inyección & \multicolumn{3}{|c|}{$25 \mathrm{ml}$} \\
\hline Detector UV/VIS & \multicolumn{3}{|l|}{$254 \mathrm{~nm}$} \\
\hline Detector fluorescencia & \multicolumn{3}{|c|}{$\begin{array}{c}\text { Excitación: } 340 \text { nm } \\
\text { Emisión: } 425 \text { nm } \\
\text { Ganancia : । }\end{array}$} \\
\hline
\end{tabular}




\section{Equipo analítico}

Para el análisis de la concentración de HPA se utilizó un cromatógrafo líquido de alta eficiencia marca DIONEX modelo ICS-3000, provisto de dos detectores acoplados en línea: ultravioletavisible y fluorescencia. Las condiciones de operación del equipo establecido para el presente método analítico se muestran en el cuadro 1.

El cromatógrafo se dejo acondicionando con la fase móvil al menos una hora antes de iniciar las corridas, hasta que la señal fue estable; luego de esto se cargaron los estándares y réplicas respectivas y se corrió el software del equipo (Chromaleon Versión 6.80 SR6).

\section{Análisis de las muestras}

Las muestras de partículas presentes en el aire se recolectan sobre filtros de cuarzo pretratados (calentados a $650{ }^{\circ} \mathrm{C}$ en una mufla durante ocho horas), los cuales se colocan en equipos muestreadores de aire de alto volumen durante 24 horas a un caudal de $1,13 \mathrm{~m} / \mathrm{min}$. Una vez finalizado el muestreo, el filtro se debe recubrir con papel aluminio para evitar la degradación de los HAP, además de ser transportado hasta el lugar de análisis a una temperatura inferior a los $4^{\circ} \mathrm{C}$.

La extracción de las muestras se lleva a cabo cortando la mitad del filtro en tiras y colocándolas en frascos de vidrio con tapa en un baño ultrasónico. Se realizan tres extracciones de 15 minutos cada una, con $15 \mathrm{ml}$ de acetonitrilo y posteriormente se concentra en rotavapor a $35-36{ }^{\circ} \mathrm{C}$, hasta un aproximado de 0.5 $\mathrm{ml}$. El concentrado resultante se trasvasa a un balón aforado de I $\mathrm{ml}$ con pipeta pasteur y se lleva a la marca de aforo, con acetonitrilo.

\section{Parámetros determinados durante la validación}

- Linealidad: para determinar el ámbito dentro del cual la respuesta del equipo se comporta de forma lineal con respecto a la concentración de los analitos de interés, se preparó, a partir de la disolución madre certificada disponible, una solución intermedia y a partir de esta una curva de calibración de 0,05 hasta 5 mg/l para todos los compuestos.

- Límites de detección y cuantificación: para establecer el nivel de concentración al cual la detección resulta problemática para los analitos de interés, se inyectó una curva de calibración del rango de trabajo y se aplicó la metodología de Meier \& Zund para poder determinar estos límites.

- Homocedasticidad/Heterocedasticidad: con el fin de determinar si la desviación estándar de los valores de " $y$ " varía significativamente a lo largo del rango de trabajo del método

Cuadro 2. Tiempos de retención de los hidrocarburos aromáticos policíclicos obtenidos por CLAE.

\begin{tabular}{|c|c|c|}
\hline Compuesto & Tiempo de retención (min) & Sensibilidad \\
\hline Naftaleno & 11.30 & UV \\
\hline Acenaftileno & 12.20 & UV \\
\hline Fluoreno & 14.20 & UV \\
\hline Acenafteno & 14.58 & UV \\
\hline Fenantreno & 15.33 & UV \\
\hline Antraceno & 16.00 & UV \\
\hline Fluoranteno & 17.507 & Emisión \\
\hline Pireno & 18.533 & Emisión \\
\hline Benzo (a) antraceno & 19.30 & Emisión \\
\hline Criseno & 20.62 & UV \\
\hline Benzo (b) fluoranteno & 23.021 & Emisión \\
\hline Benzo $(k)$ fluoranteno & 23.319 & Emisión \\
\hline Benzo (a) pireno & 23.987 & Emisión \\
\hline Dibenzo (a,h) antraceno & 24.802 & Emisión \\
\hline Benzo (g,h,i) perileno & 26.560 & UV \\
\hline Ideno (l,2,3-C.D) pireno & 27.361 & Emisión \\
\hline
\end{tabular}


Cuadro 3. Límites de detección y cuantificación de los HAP según Meier \& Zund (200l)

\begin{tabular}{|c|c|c|}
\hline Compuesto & LD $(\mathrm{mg} / \mathrm{l})$ & LC $(\mathrm{mg} / \mathrm{l})$ \\
\hline Naftaleno & 0,03 & 0,04 \\
\hline Acenaftileno & 0,02 & 0,04 \\
\hline Fluoreno & 0,03 & 0,06 \\
\hline Acenafteno & 0,06 & 0,1 \\
\hline Fenantreno & 0,03 & 0,05 \\
\hline Antraceno & 0,03 & 0,05 \\
\hline Fluoranteno & 0,03 & 0,05 \\
\hline Pireno & 0,03 & 0,06 \\
\hline Benzo (a) antraceno & 0,02 & 0,03 \\
\hline Criseno & 0,02 & 0,03 \\
\hline Benzo (b) fluoranteno & 0,04 & 0,08 \\
\hline Benzo (k) fluoranteno & 0,03 & 0,05 \\
\hline Benzo (a) pireno & 0,05 & 0,08 \\
\hline Dibenzo (a,h) antraceno & 0,06 & 0,1 \\
\hline Benzo (g,h,i) perileno & 0,03 & 0,06 \\
\hline Ideno (l,2,3-C.D) pireno & 0,05 & 0,09 \\
\hline
\end{tabular}

(comportamiento heterocedástico), en vez de tener valores que no sean significativamente distintos para todas las concentraciones (comportamiento homocedástico), se analizaron cinco réplicas independientes de patrones a tres niveles de concentración.

- Repetibilidad y reproducibilidad: para establecer la precisión del método, se analizaron cinco réplicas de una patrón intermedio durante tres días consecutivos. Se utilizaron como referencia las disposiciones de la norma ISO 5725-2: 1999.

- Exactitud: la eficiencia de recuperación se analizó mediante el enriquecimiento de cinco blancos de análisis a tres niveles de concentración diferentes. Posteriormente se preparó un gráfico de dispersión de valor experimental versus el teórico al cual se le aplicó un ajuste lineal, donde la pendiente no debía ser significativamente diferente de I y el intercepto al eje " $y$ " no debe ser significativamente diferente de 0 , si los datos fueran satisfactorios.

\section{Resultados y discusión}

Los tiempos de retención que se obtuvieron para cada compuesto según las condiciones descritas en el cuadro I, se presentan en el cuadro 2.
Como parte de la determinación de los parámetros de desempeño del método, se calculó el nivel de concentración al cual resulta ser un problema detectar estos compuestos. El límite de detección (LD) se define como la cantidad mínima de sustancia que puede ser detectada con fiabilidad por un método analítico determinado, mientras que el límite de cuantificación (LC) se reporta como la concentración más baja de analito, en una muestra que puede ser cuantitativamente determinada con cierta confianza (EURACHEM, 1998).

Ambos límites se calcularon según el método de Meier \& Zund (200 I), basándose en los límites de confianza de la curva de calibración. El cuadro 3 presenta los resultados de estos límites en $\mathrm{mg} / \mathrm{l}$, para una curva de calibración que posee un rango desde $0.05 \mathrm{mg} / \mathrm{l}$ hasta $5 \mathrm{mg} / \mathrm{l}$.

Una vez determinados los límites de detección, se preparó una curva de calibración de 0,05 mg/l a 5 mg/l, a partir de un cóctel certificado que combina los 16 hidrocarburos aromáticos policíclicos de una misma concentración, con lo que se determino el ámbito lineal del método; el área se trabaja en unidades cuentas. En la figura I se presentan las curvas de calibración obtenidas para tres HPA.

Para el ámbito de concentración de 0,05 a 5 mg/L, el método presentó una linealidad adecuada utilizando el modelo de mínimos cuadrados, con coeficientes 


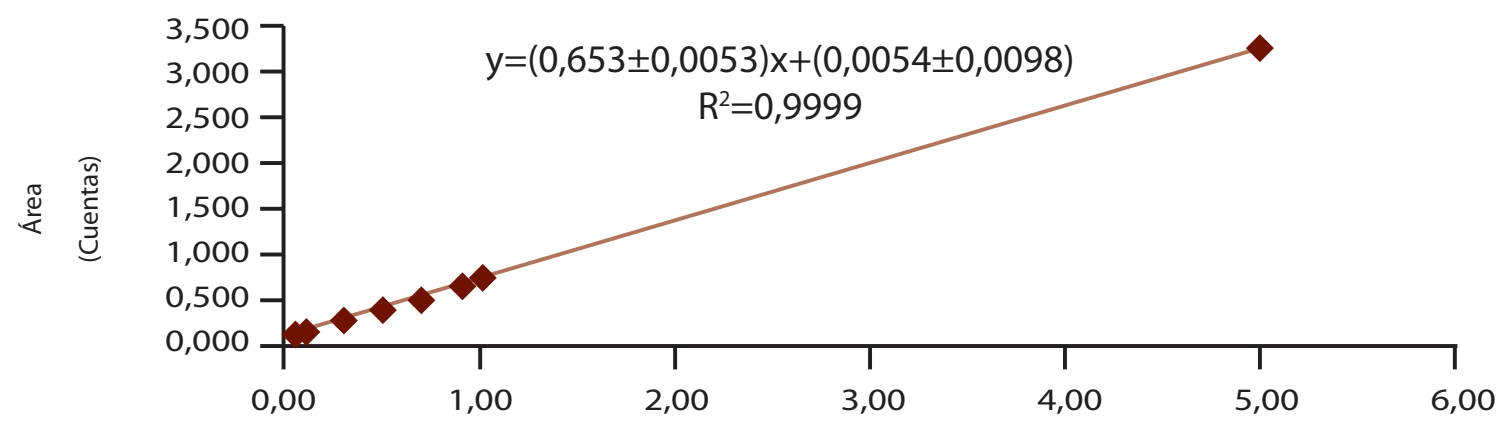

Concentración en mg/l de Naftaleno

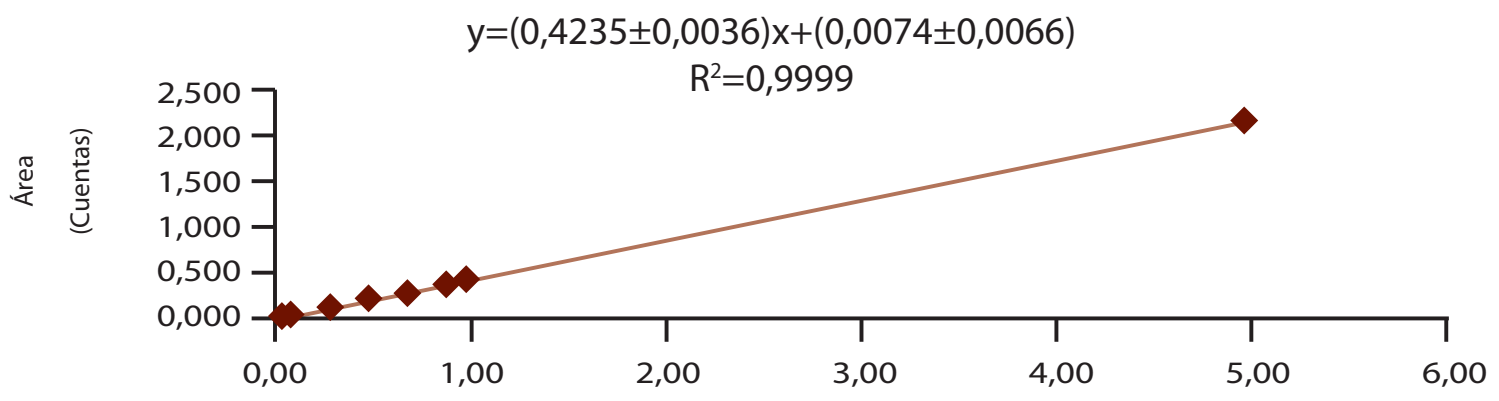

Concentración en mg/l de Acenaftileno

$$
\begin{gathered}
y=(2,9816 \pm 0,0273) x+(0,0427 \pm 0,0508) \\
R^{2}=0,9999
\end{gathered}
$$

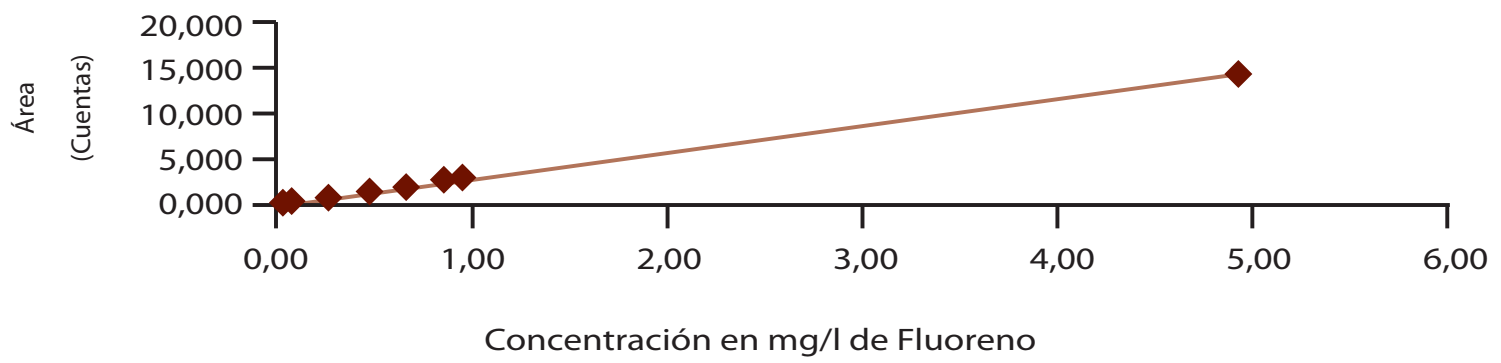

Figura I. Ámbito lineal de trabajo para tres HPA diferentes.

de correlación superiores a 0,99. Para efectos de rango de trabajo, para los análisis posteriores se utilizó el ámbito de 0, I a I mg/l.

Al utilizar una recta de regresión de " $y$ " sobre " $x$ " para realizar la cuantificación de un analito, es importante conocer si la desviación estándar de los valores de " $y$ " aumenta con la concentración del analito (comportamiento heterocedástico), en vez de tener el mismo valor para todas las concentraciones (comportamiento homocedástico), con el fin de determinar el tipo de regresión que se va a utilizar: ponderada o no ponderada.

Este comportamiento se estimó mediante la medición de cinco réplicas independientes de patrones a tres niveles de concentración. Posteriormente, para cada grupo de datos se calculó la varianza y se hizo una comparación entre ellos, mediante la aplicación de la prueba $\mathrm{F}$ de dos colas a un 95\% de nivel de confianza, 
Cuadro 4. Resultados obtenidos en el estudio del comportamiento de las desviaciones estándar de las señales para tres niveles de concentración de 16 HPA.

\begin{tabular}{|c|c|c|c|c|}
\hline \multirow[b]{2}{*}{ Compuesto } & \multicolumn{3}{|c|}{ Desviación estándar } & \multirow{2}{*}{$\begin{array}{c}\text { Comportamiento } \\
\text { de las desviaciones } \\
\text { estándar }\end{array}$} \\
\hline & $\begin{array}{c}\text { Concentración } \\
\text { Baja }\end{array}$ & $\begin{array}{c}\text { Concentración } \\
\text { Media }\end{array}$ & $\begin{array}{c}\text { Concentración } \\
\text { Alta }\end{array}$ & \\
\hline Naftaleno & 0,0004 & 0,0019 & 0,0086 & Heterocedástico \\
\hline Acenaftileno & 0,0001 & 0,0004 & 0,0082 & Heterocedástico \\
\hline Fluoreno & $0,000 \mid$ & 0,0004 & $0,008 \mid$ & Heterocedástico \\
\hline Acenafteno & 0,0001 & 0,0004 & 0,0070 & Heterocedástico \\
\hline Fenantreno & 0,0001 & 0,0005 & $0,009 \mid$ & Heterocedástico \\
\hline Antraceno & 0,0001 & 0,0005 & 0,0082 & Heterocedástico \\
\hline Fluoranteno & 0,0001 & 0,0004 & 0,0086 & Heterocedástico \\
\hline Pireno & 0,0001 & 0,0006 & 0,0080 & Heterocedástico \\
\hline $\begin{array}{l}\text { Benzo (a) } \\
\text { antraceno }\end{array}$ & 0,0001 & 0,0004 & 0,0076 & Heterocedástico \\
\hline Criseno & 0,0001 & 0,0003 & 0,0073 & Heterocedástico \\
\hline $\begin{array}{l}\text { Benzo (b) } \\
\text { fluoranteno }\end{array}$ & 0,0001 & 0,0003 & 0,0087 & Heterocedástico \\
\hline $\begin{array}{c}\text { Benzo (k) } \\
\text { fluoranteno }\end{array}$ & 0,0001 & 0,0004 & 0,0090 & Heterocedástico \\
\hline Benzo (a) pireno & 0,0002 & 0,0005 & 0,0084 & Heterocedástico \\
\hline $\begin{array}{c}\text { Dibenzo }(\mathrm{a}, \mathrm{h}) \\
\text { antraceno }\end{array}$ & 0,0001 & 0,0004 & 0,0101 & Heterocedástico \\
\hline $\begin{array}{c}\text { Benzo }(g, h, i) \\
\text { perileno }\end{array}$ & 0,0001 & 0,0003 & 0,0075 & Heterocedástico \\
\hline $\begin{array}{l}\text { Indeno (I,2,3- } \\
\text { C.D) pireno }\end{array}$ & & & & Heterocedástico \\
\hline
\end{tabular}

con el fin de determinar si al comparar un nivel de concentración contra otro existe o no diferencia significativa en la dispersión de los datos. Los tres niveles evaluados fueron: 0,I mg/l, 0,5 mg/l y I mg/l para los 16 compuestos. En el cuadro 4 se muestran los resultados obtenidos para cada compuesto.

La aplicación de la prueba $F$ en las desviaciones estándar a los diferentes niveles de concentración, reveló que las desviaciones estándar de las señales no permanecen invariantes al aumentar la concentración, por esta razón se hace necesario para el cálculo de la concentración de las muestras aplicar una regresión ponderada.

La exactitud del método se evaluó enriqueciendo cinco blancos de análisis a tres niveles de concentración, utilizando un estándar certificado de distinto lote. Al evaluar las pendientes y los interceptos para cada uno de los 16 compuestos, se puede concluir que no son significativamente diferentes a uno en el caso de las pendientes y a cero en el caso de los interceptos. Esto concluye que el método no presenta sesgos significativos para un nivel de confianza del 95\%. En la figura 2 se pueden observar las gráficas de los valores experimentales y teóricos para cuatro de los HPA.

Para determinar el componente de precisión de la exactitud, reproducibilidad y repetibilidad se analizaron cinco réplicas de un patrón intermedio $(5 \mathrm{mg} / \mathrm{l})$ durante tres días. Para realizar este estudio se siguieron las disposiciones de la norma ISO 57252: 1999 "Exactitud (veracidad y precisión) de los metodos y resultados de medición. Parte 2: Método básico para la determinación de la repetibilidad y reproductibilidad de un método de medición". En el cuadro 5 se muestran los resultados de los siguientes parámetros:

- $\mathrm{s}_{\mathrm{r}}$ : Desviación típica de la repetibilidad.

- $\mathrm{S}_{\mathrm{R}}$ : Desviación típica de la reproducibilidad.

- $r$ : Límite de repetibilidad. 

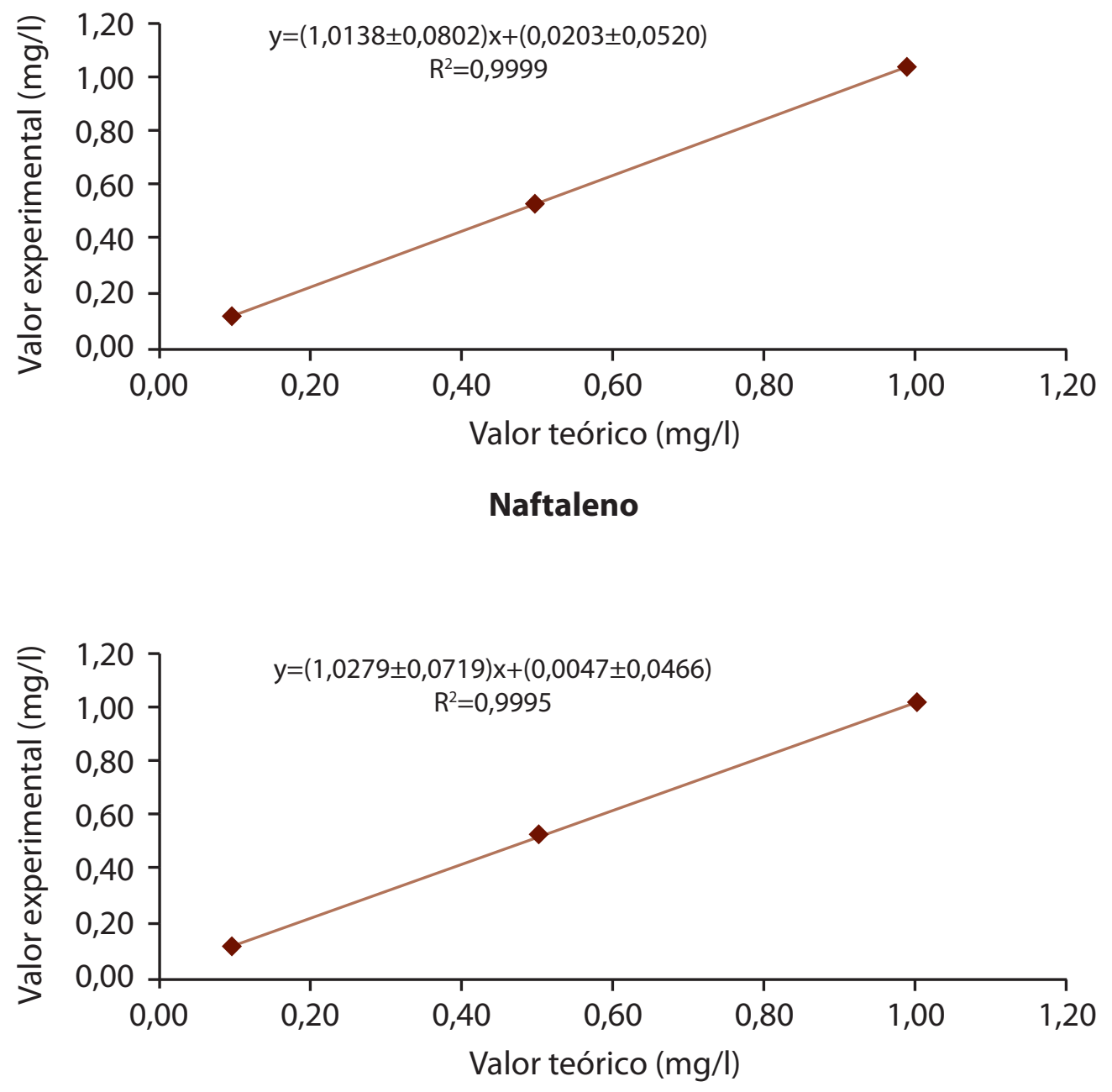

Acenaftileno

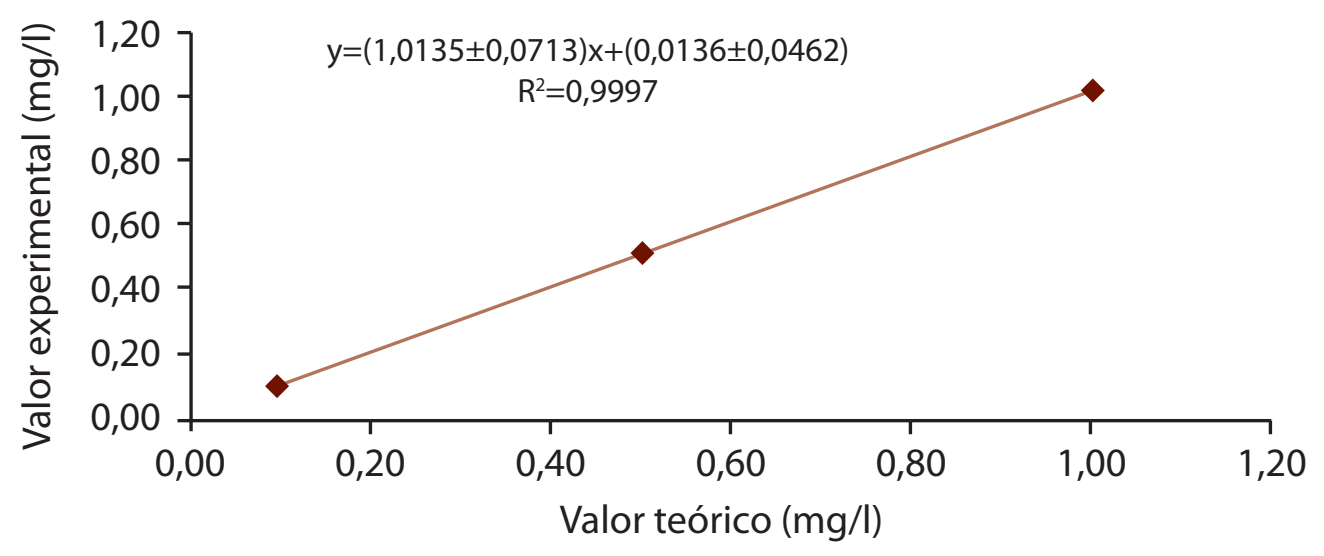

\section{Fluoreno}


Cuadro 5. Parámetros de repetibilidad y reproducibilidad del método de análisis para los 16 HPA.

\begin{tabular}{|c|c|c|c|c|c|c|}
\hline Compuesto & $S_{r(m g /)}$ & $S_{R(m g / l)}$ & $r_{(\mathrm{mg} / \mathrm{l})}$ & $R_{(\mathrm{mg} /)}$ & $\mathrm{CV}_{r}(\%)$ & $\mathrm{CV}_{\mathrm{R}}(\%)$ \\
\hline Naftaleno & 0,01 & 0,01 & 0,03 & 0,03 & 2,21 & 2,17 \\
\hline Acenaftileno & 0,02 & 0,02 & 0,05 & 0,07 & 3,32 & 4,86 \\
\hline Fluoreno & 0,02 & 0,02 & 0,05 & 0,06 & 3,16 & 4,11 \\
\hline Acenafteno & 0,02 & 0,02 & 0,04 & 0,06 & 3,01 & 4,36 \\
\hline Fenantreno & 0,06 & 0,02 & 0,05 & 0,17 & 3,00 & 11,44 \\
\hline Antraceno & 0,02 & 0,02 & 0,04 & 0,06 & 2,92 & 4,06 \\
\hline Fluoranteno & 0,02 & 0,01 & 0,04 & 0,07 & 2,77 & 4,72 \\
\hline Pireno & 0,04 & 0,02 & 0,06 & 0,10 & 4,03 & 6,78 \\
\hline Benzo (a) antraceno & 0,02 & 0,02 & 0,04 & 0,06 & 2,92 & 3,95 \\
\hline Criseno & 0,02 & 0,01 & 0,04 & 0,06 & 2,67 & 4,13 \\
\hline Benzo (b) fluoranteno & 0,02 & 0,01 & 0,04 & 0,06 & 2,82 & 4,08 \\
\hline Benzo (k) fluoranteno & 0,04 & 0,03 & 0,08 & 0,11 & 5,33 & 7,78 \\
\hline Benzo (a) pireno & 0,03 & 0,02 & 0,05 & 0,07 & 3,12 & 4,93 \\
\hline $\begin{array}{c}\text { Dibenzo }(\mathrm{a}, \mathrm{h}) \\
\text { antraceno }\end{array}$ & 0,02 & 0,02 & 0,04 & 0,05 & 3,36 & 3,21 \\
\hline Benzo $(g, h, i)$ perileno & 0,02 & 0,01 & 0,04 & 0,07 & 4,47 & 2,85 \\
\hline $\begin{array}{c}\text { Ideno }(\text { I,2,3-C.D }) \\
\text { pireno }\end{array}$ & 0,03 & 0,02 & 0,05 & 0,07 & 4,90 & 3,29 \\
\hline
\end{tabular}

- R: Límite de reproducibilidad

- $\mathrm{CV}_{\mathrm{r}}$ : Coeficiente de variación de la repetibilidad

- $\mathrm{CV}_{\mathrm{R}}$ : Coeficiente de variación de la reproducibilidad

En el cuadro anterior se observa que los coeficientes de variación en la mayoría de los casos son menores al 5\%, lo que es aceptable, para decir que el método es repetible en un mismo día y reproducible en varios días en la mayoría de los casos, esto porque por características propias del compuesto, como las del Fenatreno, el Pireno y el Benzo (k) fluoranteno, presentan coeficientes de variación de la reproducibilidad superiores al 5\%, por lo cual se concluye que estos compuestos son repetibles pero poco reproducibles.

\section{Conclusiones}

Los parámetros de desempeño obtenidos en la validación del método de análisis utilizado en la determinación de la concentración de hidrocarburos aromáticos políciclicos presentes en las partículas
PMIO y PM2.5, garantizan que este es apto para el fin propuesto, ya que tanto su sesgo como la evaluación de la precisión se encuentran entre los valores recomendados para un método analítico.

La posibilidad de utilizar dos detectores acoplados en línea (UV/Visible-Fluorescencia) permite obtener límites de detección menores a los reportados por otros métodos que utilizan cromatografía de gases con detector de masas para la cuantificación de estos compuestos.

\section{Bibliografía}

Adamson, I.Y.R.; Prieditis, H.; Hedgecock, C. \& Vincent, R. (2000). Zinc is the toxic factor in the lung response to an atmospheric particulate sample. Toxicology and Applied Pharmacology 166, I | I- I 19.

Birmili, W.; Allen, A.; Bary, F. \& Harrison, R. (2006). Trace metal concentrations and water solubility in sizefractionated atmospheric particles and influence of road traffic. Environonmental Science \& Technology 14 (4): | |44-| | 53. 
Cincinelli, A. ; Bubba, M. ; Martellini, T. ; Gambaro, A. \& Lepri, L. (2007). Gas-particle concentration and distribution of $n$-alkanes and polycyclic aromatic hydrocarbons in the atmosphere of Prato (Italy). Chemosphere 68: 472-478.

Fernández, A.; Ternero, M.; Barragandela, F. \& Jiménez, J. (2002). A chemical speciation of trace metals for fine urban particles. Atmospheric Environment 36 (5): 773780.

Figuren,A. \& Miguel, H. (2003). Determination of semivolatile and particulate polycyclic aromatic hydrocarbons in SRM 1649a and PM2.5 samples by HPLC-fluorescence. Polycyclic Aromatic Compounds 23(2): 194- 195.

Mastandrea, C.; Chichizola, C.; Ludueña, B.; Sánchez, H.; Álvarez, H. \& Gutiérrez, A. (2005). Acta Bioquímica Clínica Latinoamericana. Recuperado el 7 mayo, 2010 en http://www.scielo.org.ar/scielo.php?pid

Meier, P. \& Zund, R. (200 I). Stadistical methods in analytical chemistry. Wiley-Interscience.

Nawrot, T.; Plusquin, M.; Hogervorst, J.; Roels, H.; Celis, H.; Thijs, L.; Vangronsveld, J.; Van Hecke, E. \& Staessen, J. (2006). Environmental exposure to cadmium and risk of cancer: a prospective population-based study. Lancet 7 , | 19-126.
Ravindra, K.; Bencs, E.; Wauters, J.; Hoog, F.; Deutsch, E.; Bleux, P.; Berghmans, R. \& Grieken, C. (2006). Seasonal and site-speci.c variation in vapour and aerosol phase PAHs over Flanders (Belgium) and their relation with anthropogenic activities. Atmospheric Environment 40, 77|-778.

Richards, R. J. ( 1 997). Small particles, big problems. Biologist 44, 249-25I.

Schwartz, J. \& Meas, L.M. (2000). Fine particles are more strongly associated than coarse particles with acute respiratory health effects in schoolchildren. Epidemiology II, 6- 10 .

Seinfeld, S \& Pandis, N. (1 998). Atmospheric Chemistry and Physics. Wiley interscience, 743-744.

Sheng, P.; Peng, Y.; Chen, Z. \& Zhang, J. (2003). Distribution of particulate and vapor-phase n-alkanes and polycyclic aromatic hydrocarbons in urban atmosphere of Guangzhou, China. Atmospheric Environment 37, 289298.

Stellman,J.\&McCan,M.( 1 998).Hidrocarburospoliaromáticos. En: Enciclopedia de Salud y Seguridad en el Trabajo. 3a. edición. Madrid: Organización Internacional del Trabajo. Ministerio de Trabajo y Asuntos SocialesSubdirección General de Publicaciones. 